\title{
Validity of Addenbrooke's Cognitive Examination to Discriminate between Incipient Dementia and Depression in Elderly Patients of a Private Clinic in Lima, Peru
}

\author{
Eder Herrera-Pérez $^{a}$ Nilton Custodio ${ }^{b}$ David Lira ${ }^{b}$ Rosa Montesinos ${ }^{c}$ \\ Liliana Bendezu ${ }^{b}$ \\ a Scientific Research Projects Unit, National Institute of Child Health, ${ }^{b}$ Unit of Cognitive \\ Impairment and Dementia Prevention, Department of Neurology, Clínica Internacional, and \\ 'Unit of Rehabilitation Medicine, Clínica Internacional, Lima, Peru
}

Key Words

Dementia · Depression · Differential diagnosis · Addenbrooke's Cognitive Examination

\section{Abstract}

Background/Aims: Dementia and depression are different clinical conditions, but share common features, and can be indistinguishable in the initial disease stages. We aimed to establish whether the Peruvian version of the Addenbrooke's Cognitive Examination (ACE-Peru) can distinguish between the cognitive profile in patients with incipient dementia and that in patients with depression. Methods: This was a cross-sectional study to assess the performance on the ACE-Peru of 193 elderly subjects (102 with dementia, 21 with depression, and 70 healthy controls). Depending on the diagnosis, there were two groups of cognitive impairment $(\mathrm{CI})$ - the primary neurodegenerative (PN-CI) subtype and the secondary to depression (SD-CI) subtype - as well as a non-CI group. The area under the curve (AUC) of the receiveroperating characteristic curve was determined to compare the diagnostic performance, using the diagnosis of $\mathrm{CI}$ as the gold standard. Results: In our sample of elderly subjects aged 59-82 years with at least 7 years of education, the ACE-Peru showed a significantly better performance than the MMSE (AUC $=0.997$ vs. $A U C=0.887 ; p<0.05)$ for the discrimination between PN-CI and SD-CI. Conclusions: The ACE-Peru is able to distinguish between the cognitive profile in patients with incipient dementia and that in patients with depression. 


\section{Introduction}

Dementia is considered to be one of the main geriatric problems due to impaired functionality and independence [1]. The estimated prevalence of senile dementia in Latin America is $8.5 \%$ among people older than 60 years [2-8]. There is also an increase in new therapeutic options [9]. For this reason diagnostic tools which make early case detection possible need to be available. Thus, serial instruments have been developed, such as the Mini-Mental State Examination (MMSE) [10], the Mental Cambridge Disorders of the Elderly Examination (CAMDEX) [11-13], and its cognitive component, the Cambridge Cognitive Examination (CAMCOG) [14], as well as the Dementia Rating Scale (DRS) [15-18].

Although the MMSE is the instrument most frequently used, it lacks sufficient sensitivity to detect mild impairment observed in the early stages of Alzheimer's dementia (AD) as well as other changes in frontotemporal dementia (FTD) [19]. Moreover, CAMCOG, DRS, and the neuropsychological assessment [ideal instruments to assess cognitive impairment (CI)] are complex and need highly trained personnel and specialized equipment [20], which is usually not available at the primary care level. The Addenbrooke's Cognitive Examination (ACE), which is simpler and does not need complex equipment, could be performed at the primary care level. In other studies, it has shown a promising diagnostic performance. Thus, it is a valuable alternative in cases of early detection of dementia by general practitioners.

Depression is another common problem [21] in the elderly population [21], affecting between 13 and $18 \%$ of people older than 65 years [22, 23] and up to $40 \%$ of patients with comorbidities [24, 25]. More than half of the cases of depression late in life are secondary to the consumption of some legal drugs (depression secondary to drugs) or to the coexistence of another underlying medical condition (depression secondary to medical conditions) such as CI [22]. Dementia and depression often have various common features: (1) patients with dementia can present depressive symptomatology, (2) patients with depression can present significant CI (i.e. 'pseudodementia'), and (3) depression can be an early reaction to any CI, an early symptom of dementia, or a risk factor for dementia [26]. In some cases it is therefore difficult to establish whether the $\mathrm{CI}$ is secondary to an incipient organic process, as observed in $\mathrm{AD}$, or to an affective disorder such as depression [27]. The staging becomes even more complicated in case of the co-occurrence of both conditions, which cannot be distinguished from one another $[26,28]$. Thus, the differential diagnosis of dementia and depression represents one of the most frequent clinical problems [28].

This problem is particularly relevant in the practice of primary health care, since it has been observed that nonspecialist medical doctors do not recognize up to $21 \%$ of the cases of dementia, while $20 \%$ of nondemented subjects are misdiagnosed as having dementia [29]; in fact, only $50 \%$ of the patients with dementia are detected by medical professionals [30]. This reveals that general practitioners need diagnostic tools which allow dementia screening at the early stages of disease and which are easily applicable in the daily practice in primary care centers.

Previously, the performance of the ACE to discriminate between dementia and depression has been evaluated, although the results have been contradictory [31-34]. The following factors still need to be established: the utility of the Peruvian version of the ACE (ACE-Peru) for these purposes, the appropriate cutoff value, and the need for its adjustment for variables such as gender, age, and years of education.

This study assesses the validity of the ACE-Peru in discriminating between the CI associated with depression and the $\mathrm{CI}$ secondary to incipient dementia in patients older than 55 years, as well as in discriminating between the CI secondary to depression and the absence of CI. Additionally, the performance of the ACE-Peru was compared with the MMSE based on each test performance evaluated against the gold standard. 
Herrera-Pérez et al.: Validity of Addenbrooke's Cognitive Examination to Discriminate between Incipient Dementia and Depression in Elderly Patients of a Private Clinic

\section{Materials and Methods}

\section{Design of the Study}

This is a secondary analysis of the database derived from the implementation of a guideline-based diagnostic protocol for dementia in the Service of Neurology of a private clinic in Lima, Peru. Previously, this database has been partially used for the validation of the ACE-Peru [35].

\section{Population and Sample}

For this analysis we included the data of patients older than 55 years, who had Spanish as their native language, who had at least 6 years of education, and who visited the Neurology Consulting Room during the period between April 2006 and April 2009. The cases were selected among those patients who reported cognitive problems (subjective cognitive impairment, SCI) that were confirmed for an applied screening test (objective cognitive impairment, OCI). Healthy subjects were selected among the relatives of non-SCI and non-OCI patients.

We excluded those subjects with structural and/or functional deficits (visual or auditory deficits) that affect their performance and interfere with the carrying out of the cognitive tests, subjects with a history of diseases associated with secondary CI [e.g. cerebrovascular illness, hypothyroidism, and central nervous system infections (HIV or syphilis), severe encephalic traumatism, and subdural hematoma], subjects with a history of conditions associated with secondary CI (e.g. vitamin B12 deficiency, chronic hepatopathy, or nephropathy, and addiction or abuse of substances), as well as subjects with a score of more than 4 on the Hachinski index (which suggests underlying cerebrovascular deficits).

\section{Procedures}

According to the diagnostic protocol (the gold standard for this study), first CI screening was carried out to establish the cognitive status. Second, the etiologic diagnosis of CI was assessed and also the diagnosis of depression, whenever dementia was found to be absent. Finally, the classification of dementia was established in demented patients. Both the tests of interest and the gold standard were systematically and independently assessed in all consecutive eligible patients.

\section{Stage 1: Screening}

CI screening was carried out by means of the application of the questionnaire for the assessment of subjective memory complaints [36], the MMSE [10] and the Clock-Drawing Test, Manos' version [37]. The Pfeffer Functional Activities Questionnaire (PFAQ) was used for functional evaluation [38]. Those patients with at least one positive test in CI screening were classified as OCI.

Stage 2: Etiologic Diagnosis

Clinical assessment including Beck's Depression Inventory (BDI), laboratory tests, and brain CT scans/magnetic resonance neurography were applied to investigate the main causes of $\mathrm{CI}$, so as to ascertain whether patients had dementia, depression, or another condition. For those patients who presented some cause of SCI, appropriate treatment was offered. When we observed a posttherapeutic $\mathrm{CI}$, the patients were classified as secondary $\mathrm{CI}$, like those patients with medical conditions where $\mathrm{CI}$ is naturally irreversible. Those patients, in whom no specific cause of CI was identified, were classified as primary CI. 
Stage 3: Dementia Subtype Diagnosis

All patients with primary CI were submitted sequentially to the neurological and neuropsychological evaluation for the dementia diagnosis, which was carried out by a team of professionals including two neurologists and one neuropsychologist.

The neurologists performed an integral neurological evaluation and applied the neuropsychiatric battery: Neuropsychiatric Inventory (NPI). The neuropsychologist applied a battery which consisted of the following 9 tests: Rey Auditory Verbal Learning Test, Logical Memory Subtest of Wechsler Memory Scale-Revised, Trail Making Test A and B, Rey's Complex Figure, Test of Denomination of Boston, Wisconsin Card Sorting Test, Letter-Number (Wechsler Adult Intelligent Scale III Subtest), and Digit Span.

The definitive dementia diagnosis (dementia subtype) was based on a consensus between the professional team members according to appropriate diagnostic criteria. The applied criteria were the following: (1) Diagnostic and Mental Statistical Manual of Disorders, 4th Edition, Text Revision (DSM-IV-TR) for the dementia diagnosis, (2) National Institute of Neurologic, Communicative Disorders and Stroke-Alzheimer's Disease and Related Disorders Association (NINCDS-ADRDA) for the AD diagnosis, (3) consensus of Neary for the FTD diagnosis, (4) United Kingdom Parkinson's Disease Society Brain Bank (UK-PDSBB) for the diagnosis of Parkinson's disease (PD), and (5) American Academy of Neurology (AAN) for the diagnosis of mild CI (MCI). Although MCI is not a dementia subtype, it has been recognized as a dementia precursor [39].

We used the term primary neurodegenerative $\mathrm{CI}$ (PN-CI) for the demented patients without considering those with predementia (MCI). The cases of CI secondary to depression (SD-CI) were defined as patients with secondary CI; those with medical causes of CI (other than depression) or with a BDI value suggesting depression (BDI $\geq 13$ points) were excluded. The group of cognitively healthy subjects was selected from the healthy relatives of the patients, in whom we applied the same diagnostic protocol for CI to exclude subjects with CI.

All evaluations were done blinded and independently by one neurologist who applied the ACE to all the participants at the end of stage 1 (screening), before Beck's test application, and long before the definitive establishment of the diagnosis. For this study we included patients with incipient organic dementia (AD, FTD, and PD dementia), patients with depression, and subjects without CI (healthy control group).

\section{Statistical Analysis}

Sociodemographic variables were compared with $\chi^{2}$ and Mann-Whitney tests for pairs of groups (PN-CI vs. SD-CI and SD-CI vs. healthy group). Sensitivity and specificity of the ACE and the MMSE were calculated.

As both the ACE and the MMSE are continuous variables, we sought to assess their diagnostic performance for different threshold values. For this we estimated the area under the curve (AUC) for the receiver-operating characteristic (ROC) curves for the ACE and the MMSE. The analysis was performed by using STATA 12.0 (Stata Corp., College Station, Tex., USA).

\section{Ethical Aspects}

This study was authorized by the Investigation and Teaching Unit of the Clínica Internacional. The present study was approved by the Ethics Committee of the Universidad Peruana Cayetano Heredia. 
Table 1. Demographic characteristics and test scores in 193 subjects attending the Neurology Consulting Room of the Clínica Internacional

\begin{tabular}{llllll}
\hline & $\begin{array}{l}\text { SD-CI } \\
\text { (depression } \\
\text { group; } \mathrm{n}=21)\end{array}$ & $\begin{array}{l}\text { PN-CI } \\
\text { (dementia } \\
\text { group; } \mathrm{n}=102)\end{array}$ & $\begin{array}{l}\text { Non-CI } \\
\text { (healthy } \\
\text { group; } \mathrm{n}=70)\end{array}$ & $\begin{array}{l}\text { p value } \\
\text { (SD-CI vs. } \\
\text { PN-CI) }\end{array}$ & $\begin{array}{l}\text { p value } \\
\text { (SD-CI vs. } \\
\text { non-CI) }\end{array}$ \\
\hline Males & $10(47 \%)$ & $49(48 \%)$ & $36(51 \%)$ & $0.972^{\mathrm{a}}$ & $0.759^{\mathrm{a}}$ \\
Age, years & $66(59-72)$ & $69(61-82)$ & $68(62-80)$ & $0.002^{\mathrm{b}}$ & $0.018^{\mathrm{b}}$ \\
Education, years & $10(8-16)$ & $10(7-18)$ & $11(7-18)$ & $0.470^{\mathrm{b}}$ & $0.364^{\mathrm{b}}$ \\
MMSE score & $27(25-29)$ & $23(16-29)$ & $29(27-30)$ & $<0.001^{\mathrm{b}}$ & $<0.001^{\mathrm{b}}$ \\
ACE score & $88(83-92)$ & $71(56-84)$ & $94(89-98)$ & $<0.001^{\mathrm{b}}$ & $<0.001^{\mathrm{b}}$ \\
\hline
\end{tabular}

Data are presented as median (range) unless indicated otherwise. ${ }^{a} \mathrm{p}$ value for the $\chi^{2}$ test. ${ }^{\mathrm{b}} \mathrm{p}$ value for the Mann-Whitney test.

\section{Results}

A total of 193 subjects were included, of whom 102 were patients with PN-CI (53 with AD, 26 with FTD, and 23 with dementia for PD), 21 were patients with SD-CI, and 70 were cognitively healthy subjects.

Significant differences were observed by age, ACE, and MMSE values between the subjects with PN-CI and healthy subjects compared to those with SD-CI (table 1). This is critical because the age is recognized as an influential factor on the cognitive performance, which is evaluated by these tests. Thus, lower values are expected in the ACE or the MMSE as the age increases.

Accordingly, we observed that the age is significantly lower between the patients with SD-CI compared to other groups. This could influence the ACE and the MMSE values, which are observed to be significantly higher in patients with PN-CI (who were oldest). Nevertheless, this significance cannot only be explained by age, since the patients with SD-CI had lower values than the healthy subjects, although the former were older. No significant differences were observed by sex and years of education.

The ACE showed a high precision in discriminating between PN-CI and SD-CI (AUC = $0.997 ; 95 \%$ confidence interval $0.99-1.00$ ) and in discriminating between SD-CI and non-CI (AUC $=0.948 ; 95 \%$ confidence interval 0.90-0.99). Although the ACE had a significantly better performance than the MMSE in discriminating between PN-CI and SD-CI (fig. 1), there were no similar performances in discriminating between SD-CI and non-CI (fig. 2).

Regarding the clinical implications, in every patient with CI in a similar context, the application of the ACE will allow to establish whether CI is caused by dementia or depression with an almost perfect precision, at high sensitivity and specificity rates $(90$ and 100\%, respectively), using a cutoff value of at least 85 points. The use of this cutoff value results in $98.37 \%$ of correctly classified subjects (table 2).

\section{Discussion}

This study aimed to assess the utility of the ACE-Peru in order to discriminate between dementia and depression in patients with $\mathrm{CI}$, showing a high discriminative performance. We found a high sensitivity (90\%) and a perfect specificity (100\%) when the ACE was applied using a cutoff value of 85 . These findings are consistent with those reported previously by other studies [32-34]. 
Dementia

Cognitive Disorders

Table 2. Cutoff values and diagnostic utility of ACE and MMSE to discriminate between SD-CI and PN-CI in 193 subjects attending the Neurology Consulting Room of the Clínica Internacional
Fig. 1. ROC curves for the discrimination between SD-CI and PN-CI by means of the ACE in comparison to the MMSE in 193 subjects attending the Neurology Consulting Room of the Clínica Internacional.
Dement Geriatr Cogn Disord Extra 2013;3:333-341

DOI: 10.1159/000354948

(C) 2013 S. Karger AG, Base www.karger.com/dee

Herrera-Pérez et al.: Validity of Addenbrooke's Cognitive Examination to Discriminate between Incipient Dementia and Depression in Elderly Patients of a Private Clinic

\section{ACE}

MMSE

Discrimination between patients with $S D-C I$ and patients with $P N-C I$ Optimal cutoff value ${ }^{\mathrm{a}}$

Sensitivity

Specificity

$85 / 86$

$27 / 28$

Specificity

0.90

0.67

Area under the curve

1.00

0.88

Discrimination between non-CI subjects and patients with $S D-C I$ Optimal cutoff value ${ }^{\mathrm{a}}$

Sensitivity

Specificity

90/91

$28 / 29$

Area under the curve

0.67

0.67

$0.948 \quad 0.895$

a The cutoff value is based both on sensitivity and specificity to obtain a maximum percentage of correct classification.

b Significant difference with regard to the MMSE, $p<0.05$. Gold standard: final diagnosis achieved with the application of the diagnostic protocol for CI.

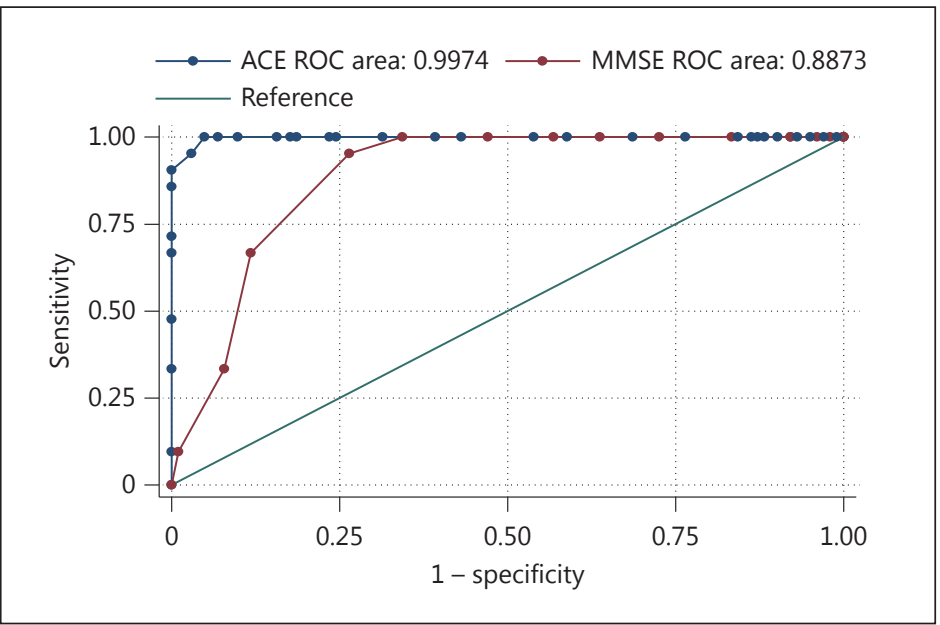

Fig. 2. ROC curves for the discrimination between SD-CI and non-CI by means of the ACE in comparison to the MMSE in 193 subjects attending the Neurology Consulting Room of the Clínica Internacional.

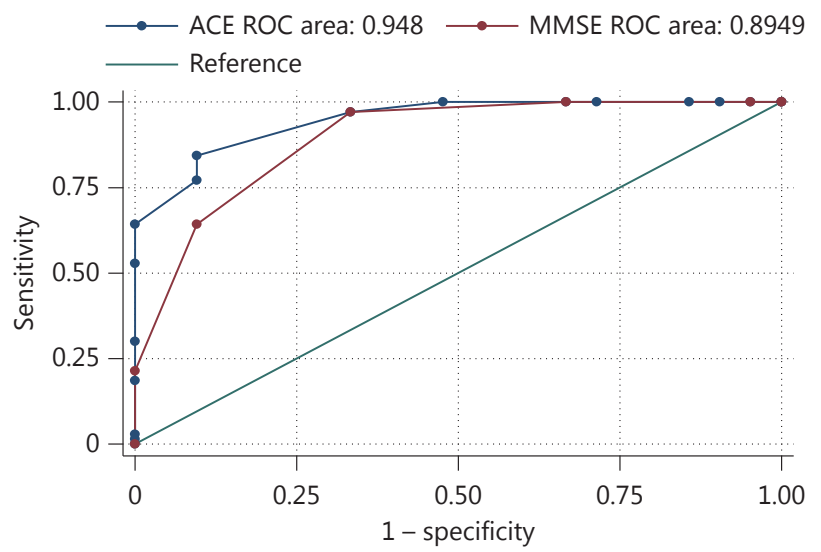


Dementia is defined as the presence of CI of two or more cognitive domains [memory and other cognitive domain(s)] [40]. Depression is defined as the absence of positive affectivity and low mood [41]. Although dementia and depression are different clinical entities, they share common features: (1) impairment of attention and working memory, (2) changes in sleep patterns, and (3) reduction of social and work-related functionality [42]. These problems have led to mistakes in medical practice. Thus, a study has found that at least 26 of every 100 patients were misdiagnosed as having dementia, 15 of whom were patients with nonrecognized depression [27]. Similarly, it is possible that some patients with depression could be misdiagnosed as having dementia, resulting in a subdiagnosis of depression. This situation has not been previously studied.

Although the cutoff value of 85 sacrifices sensitivity to maximize specificity, we believe it is appropriate because of the need to prioritize the ability of the ACE to rule out a depressive disease (suggesting a dementia disease) in every elderly patient with CI. The importance of this tool in primary health care is potentially valuable for the early detection of demented patients and their timely referral to specialized centers. Finally, the identification of patients with CI truly secondary to depressive disease is highly important for the prognosis, because appropriate treatment of depression in SD-CI patients can improve the quality of life and can even achieve a full resolution of the CI [31].

The ACE-Peru is able to distinguish between the cognitive profile in patients with incipient dementia and that in those with depression, but not between the cognitive profile in patients with depression and cognitively healthy subjects. We recommended the use of 85 as a cutoff value for differentiating between these two medical conditions in elderly patients with CI.

It was not possible to use the best possible instruments for the measurement processes, limiting the evaluation only to data previously obtained for other purposes. Thus, the diagnosis of depression was not reached by means of the criteria listed by the International Classification of Diseases Version 10 (ICD-10) or by the DSM-IV-TR. However, we used a depression indicator (BDI), which was validated in the elderly population [43] and which is particularly useful in subjects with CI [44] such as in our study population.

Finally, previous studies [31-34] did not assess the utility of the ACE in patients with PD for our study objectives. We propose a new use for this screening test: a comprehensive and simple tool in order to discriminate between dementia and depression in elderly patients with $\mathrm{CI}$, with an easy and quick application, which might be used in primary health care. We recommend assessing this application at the community level in prospective populationbased studies.

\section{Acknowledgements}

We gratefully acknowledge the support provided by Dr. Fredy Canchihuaman, who reviewed and commented on this paper.

\section{Disclosure Statement}

The authors report no conflicts of interest. 
Herrera-Pérez et al.: Validity of Addenbrooke's Cognitive Examination to Discriminate between Incipient Dementia and Depression in Elderly Patients of a Private Clinic

\section{References}

1 Ayuso Blanco T, Ederra Miranda MJ, Manubens Bertrán J, Nuin Villanueva MÁ, Villar Casado D, Zubicoa Ventura J: Abordaje de la demencia. Guía de actuación en la coordinación Atención Primaria-Neurología (Internet). Servicio Navarro de Salud - Osasunbidea, 2007 (cited Dec 12, 2012). http://www.navarra.es/NR/ rdonlyres/90E6356A-73C3-4CA1-9291-834501AA4324/0/Guiaabordajedelademencia.pdf.

2 Custodio N, García A, Montesinos R, Escobar J, Bendezú L: Prevalencia de demencia en una población urbana de Lima-Perú: estudio puerta a puerta. An Fac Med 2008;69:233-238.

-3 Ferri CP, Prince M, Brayne C, Brodaty H, Fratiglioni L, Ganguli M, et al: Global prevalence of dementia: a Delphi consensus study. Lancet 2005;366:2112-2117.

-4 Herrera E Jr, Caramelli P, Silveira ASB, Nitrini R: Epidemiologic survey of dementia in a community-dwelling Brazilian population. Alzheimer Dis Assoc Disord 2002;116:103-108.

-5 Kalaria RN, Maestre GE, Arizaga R, Friedland RP, Galasko D, Hall K, et al: Alzheimer's disease and vascular dementia in developing countries: prevalence, management, and risk factors. Lancet Neurol 2008;7:812-826.

-6 Llibre JJ, Guerra MA, Pérez-Cruz H, Bayarre H, Fernández-Ramírez S, González-Rodríguez M, et al: Dementia syndrome and risk factors in adults older than 60 years old residing in Habana (in Spanish). Rev Neurol 1999; 29:908-911.

7 Nitrini R, Bottino CMC, Albala C, Custodio Capuñay NS, Ketzoian C, Llibre Rodriguez JJ, et al: Prevalence of dementia in Latin America: a collaborative study of population-based cohorts. Int Psychogeriatr 2009;21: 622-630.

8 World Health Organization: Dementia: a public health priority (Internet). WHO, 2012 (cited Dec 4, 2012). http://www.who.int/mental_health/publications/dementia_report_2012/en/index.html.

-9 Cummings JL, Doody R, Clark C: Disease-modifying therapies for Alzheimer disease: challenges to early intervention. Neurology 2007;69:1622-1634.

10 Folstein MF, Folstein SE, McHugh PR: 'Mini-mental state'. A practical method for grading the cognitive state of patients for the clinician. J Psychiatr Res 1975;12:189-198.

11 Roth M, Tym E, Mountjoy CQ, Huppert FA, Hendrie H, Verma S, et al: CAMDEX. A standardised instrument for the diagnosis of mental disorder in the elderly with special reference to the early detection of dementia. Br J Psychiatry 1986;149:698-709.

12 Hendrie HC, Hall KS, Brittain HM, Austrom MG, Farlow M, Parker J, et al: The CAMDEX: a standardized instrument for the diagnosis of mental disorder in the elderly: a replication with a US sample. J Am Geriatr Soc 1988;36:402-408.

13 Neri M, Roth M, De Vreese LP, Rubichi S, Finelli C, Bolzani R, et al: The validity of informant reports in assessing the severity of dementia: evidence from the CAMDEX interview. Dement Geriatr Cogn Disord 1998;9:56-62.

$\checkmark 14$ Huppert FA, Brayne C, Gill C, Paykel ES, Beardsall L: CAMCOG - a concise neuropsychological test to assist dementia diagnosis: socio-demographic determinants in an elderly population sample. Br J Clin Psychol 1995; 34(Pt 4):529-541.

15 Gardner R Jr, Oliver-Muñoz S, Fisher L, Empting L: Mattis Dementia Rating Scale: internal reliability study using a diffusely impaired population. J Clin Neuropsychol 1981;3:271-275.

16 Shay KA, Duke LW, Conboy T, Harrell LE, Callaway R, Folks DG: The clinical validity of the Mattis Dementia Rating Scale in staging Alzheimer's dementia. J Geriatr Psychiatry Neurol 1991;4:18-25.

17 Smith GE, Ivnik RJ, Malec JF, Kokmen E, Tangalos E, Petersen RC: Psychometric Properties of the Mattis Dementia Rating Scale. Assessment 1994;1:123-132.

18 Vitaliano PP, Breen AR, Russo J, Albert M, Vitiello MV, Prinz PN: The clinical utility of the dementia rating scale for assessing Alzheimer patients. J Chronic Dis 1984;37:743-753.

19 Strauss EH, Sherman EMS, Spreen O: A Compendium of Neuropsychological Tests: Administration, Norms, and Commentary. Oxford, Oxford University Press, 2006.

20 Gifford DR, Cummings JL: Evaluating dementia screening tests: methodologic standards to rate their performance. Neurology 1999;52:224-227.

21 Halverson J, Bhalla R, Moraille-Bhalla P: Depression (Internet). Medscape Ref 2011 (cited Dec 4, 2012). http:// emedicine.medscape.com/article/286759-overview\#a0156.

22 Blazer D, Williams CD: Epidemiology of dysphoria and depression in an elderly population. Am J Psychiatry 1980;137:439-444.

23 Copeland JR, Gurland BJ, Dewey ME, Kelleher MJ, Smith AM, Davidson IA: Is there more dementia, depression and neurosis in New York? A comparative study of the elderly in New York and London using the computer diagnosis AGECAT. Br J Psychiatry 1987;151:466-473.

24 Koenig HG, George LK, Peterson BL, Pieper CF: Depression in medically ill hospitalized older adults: prevalence, characteristics, and course of symptoms according to six diagnostic schemes. Am J Psychiatry 1997;154: 1376-1383.

25 Burke WJ, Wengel SP: Late-life mood disorders. Clin Geriatr Med 2003;19:777-797, vii.

26 Byers AL, Yaffe K: Depression and risk of developing dementia. Nat Rev Neurol 2011;7:323-331.

27 Garcia CA, Reding MJ, Blass JP: Overdiagnosis of dementia. J Am Geriatr Soc 1981;29:407-410.

28 Reding M, Haycox J, Blass J: Depression in patients referred to a dementia clinic. A three-year prospective study. Arch Neurol 1985;42:894-896. 
Herrera-Pérez et al.: Validity of Addenbrooke's Cognitive Examination to Discriminate between Incipient Dementia and Depression in Elderly Patients of a Private Clinic

29 Barrett JJ, Haley WE, Harrell LE, Powers RE: Knowledge about Alzheimer disease among primary care physicians, psychologists, nurses, and social workers. Alzheimer Dis Assoc Disord 1997;11:99-106.

-30 Boise L, Camicioli R, Morgan DL, Rose JH, Congleton L: Diagnosing dementia: perspectives of primary care physicians. Gerontologist 1999;39:457-464.

-31 Roca M, Torralva T, López P, Marengo J, Cetkovich M, Manes F: Differentiating early dementia from major depression with the Spanish version of the Addenbrooke's Cognitive Examination (in Spanish). Rev Neurol 2008;46:340-343.

-32 Mathuranath PS, Nestor PJ, Berrios GE, Rakowicz W, Hodges JR: A brief cognitive test battery to differentiate Alzheimer's disease and frontotemporal dementia. Neurology 2000;55:1613-1620.

33 Dudas RB, Berrios GE, Hodges JR: The Addenbrooke's Cognitive Examination (ACE) in the differential diagnosis of early dementias versus affective disorder. Am J Geriatr Psychiatry 2005;13:218-226.

34 Sarasola D, de Luján-Calcagno M, Sabe L, Crivelli L, Torralva T, Roca M, et al: Validity of the Spanish version of the Addenbrooke's Cognitive Examination for the diagnosis of dementia and to differentiate Alzheimer's disease and frontotemporal dementia (in Spanish). Rev Neurol 2005;41:717-721.

35 Custodio N, Lira D, Montesinos R, Gleichgerrcht E, Manes F: Usefulness of the Addenbrooke's Cognitive Examination (Spanish version) in Peruvian patients with Alzheimer's disease and frontotemporal dementia (in Spanish). Vertex 2012;23:165-172.

36 Mías CD, Sassi M, Masih ME, Querejeta A, Krawchik R: Mild cognitive impairment: a prevalence and sociodemographic factors study in the city of Córdoba, Argentina (in Spanish). Rev Neurol 2007;44:733-738.

37 Custodio N, García A, Montesinos R, Lira D, Bendezú L: Validation of the clock drawing test - Manos' version - as a screening test for detection of dementia in older persons of Lima, Peru (in Spanish). Rev Peru Med Exp Salud Pública 2011;28:29-34.

38 Pfeffer RI, Kurosaki TT, Harrah CH Jr, Chance JM, Filos S: Measurement of functional activities in older adults in the community. J Gerontol 1982;37:323-329.

39 Panza F, Frisardi V, Capurso C, D’Introno A, Colacicco AM, Imbimbo BP, et al: Late-life depression, mild cognitive impairment, and dementia: possible continuum? Am J Geriatr Psychiatry 2010;18:98-116.

40 American Psychiatric Association: Diagnostic and Statistical Manual of Mental Disorders, Fourth Edition: DSM-IV-TR ${ }^{\circledR}$. Washington, American Psychiatric Publ., 2000.

41 García-Herrera Pérez-Bryan JM, Nogueras Morillas EV, Muñoz Cobos F, Morales Asencio JM: Guía de práctica clínica para el tratamiento de la depresión en atención primaria (Internet). 2011: Grupo para el estudio de la depresión en atención primaria (GAEDAP) (cited Dec 4, 2012). http://portal.guiasalud.es/web/guest/ catalogo?p_p_id=EXT_7_INSTANCE_mjrc\&p_p_lifecycle=0\&p_p_state=maximized\&p_p_mode=view\&_EXT_7_ INSTANCE_mjrc_struts_action=\%2Fext $\% 2 \mathrm{Fespecifico} \% 2 \mathrm{Fvista} P$ Previa\&_EXT_7_INSTANCE_mjrc_ contenidoId=80081\&_EXT_7_INSTANCE_mjrc_version=1.4.

42 Steffens DC, Potter GG: Geriatric depression and cognitive impairment. Psychol Med 2008;38:163-175.

43 Lasa L, Ayuso-Mateos JL, Vázquez-Barquero JL, Díez-Manrique FJ, Dowrick CF: The use of the Beck Depression Inventory to screen for depression in the general population: a preliminary analysis. J Affect Disord 2000;57: 261-265.

44 Silverberg NB, Ryan LM, Carrillo MC, Sperling R, Petersen RC, Posner HB, et al: Assessment of cognition in early dementia. Alzheimers Dement 2011;7:e60-e76. 